\title{
ADAPTIVE FEEDBACK LINEARIZATION CONTROL FOR ASYNCHRONOUS MACHINE WITH NONLINEAR FOR NATURAL DYNAMIC COMPLETE OBSERVER
}

\author{
Abderrahim Bentaallah* _ Ahmed Massoum * \\ Farid Benhamida ${ }^{* *}$ - Abdelkader Meroufel ${ }^{*}$
}

\begin{abstract}
This paper studies the nonlinear adaptive control of an induction motor with natural dynamic complete nonlinear observer. The aim of this work is to develop a nonlinear control law and adaptive performance for an asynchronous motor with two main objectives: to improve the continuation of trajectories and the stability, robustness to parametric variations and disturbances rejection. This control law will independently control the speed and flux into the machine by restricting supply. A complete nonlinear observer for dynamic nature ensuring closed loop stability of the entire control and observer has been developed. Several simulations have also been carried out to demonstrate system performance.
\end{abstract}

Ke yw ords: f feedback linearization, adaptive control, parameter estimation, nonlinear observer (NLO), induction motor (IM)

\section{INTRODUCTION}

The nonlinear control based on the technique of linearization within the meaning of the input-outputs proved reliable on the level of the control of the asynchronous machine [2-7]. It was demonstrated that the nonlinear controller is adept nonlinearities and maintaining its performance in a wide operating range long and as long as the machine parameters are not changed. However, it completely loses its performance when the model of the machine is subject to uncertainties in the parameters. In the case of the induction machine, the uncertain parameters are mainly the stator and rotor resistance (depending on temperature), inductors (which depend on the level of saturation), the moment of inertia and load torque (which are difficult to quantify). In addition, by examining the model, one can notice that two of these parameters fall in a linear fashion in the model (resistance and load torque) while the other two returned from a nonlinear fashion (inductors and timing of inertia). At first, researchers tried to solve this problem by developing algorithms for identification of uncertain parameters that tend to change during operation [8]. However, despite the results obtained, they were discarded because of their complexity. Parallel to this, many researchers have been developing methodologies for adaptive nonlinear linearization technique combining with adaptive methods [9-14]. All these methods have led to satisfactory results, but they limit the model structures and how they depend on uncertain parameters. Indeed, most methods proposed in the literature are for the case where the uncertain parameters of a linear fit in the model [15]. The algorithm that we propose to apply in our case is the one proposed in [1]. This algorithm is not limited to the case where the uncertain parameters of linear fit in the model but it also applies to models with uncertain parameters returned from a nonlinear fashion. In this article we present the principle of the linearization adaptive input-output intended for the control of the asynchronous machine or the parameters return in a linear way in the model of the machine (resistances and the torque of load). We then used a nonlinear observer of flux and current. The performances of the nonlinear controller adaptive will be discussed by digital simulation.

\section{ADAPTIVE NONLINEAR CONTROL OF IM}

In this section, one carries out an adaptive nonlinear order which ensures the regulation the speed and the flux of the asynchronous machine as well as decoupling between the latter. In this part, one will hold account only parameters which return in a linear way in the model of the machine. It is about rotor resistance $R_{r}$ and the torque of load $T_{L}$. To be done, one starts by designing a controller based on the technique of linearization within the meaning of the input-output applied to the nominal model, then one calculation the law of adaptation which will make it possible to estimate the vector of the dubious parameters $[8,9]$.

The dynamics of an induction motor under the assumptions of equal mutual inductances and linear mag-

\footnotetext{
* Department of Electrical Engineering, Faculty of Science Engineering, Djillali Liabès University of Sidi Bel-Abbès, Algeria ICEPS Laboratory. a_bentaallah@yahoo.fr ${ }^{* *}$ IRECOM Laboratory; farid.benhamida@yahoo.fr
} 
netic are given by the fifth-order model.

$$
\begin{aligned}
\frac{\mathrm{d} x_{1}}{\mathrm{~d} t} & =-\lambda x_{1}+\frac{k}{T_{r}} x_{3}+p k x_{4} x_{5}+\frac{1}{\sigma} u_{s \alpha}, \\
\frac{\mathrm{d} x_{2}}{\mathrm{~d} t} & =-\lambda x_{2}+\frac{k}{T_{r}} x_{4}+p k x_{3} x_{5}+\frac{1}{\sigma} u_{s \beta}, \\
\frac{\mathrm{d} x_{3}}{\mathrm{~d} t} & =\frac{M}{T_{r}} x_{1}-\frac{1}{T_{r}} x_{3}+p x_{4} x_{3}, \\
\frac{\mathrm{d} x_{4}}{\mathrm{~d} t} & =\frac{M}{T_{r}} x_{2}-\frac{1}{T_{r}} x_{4}+p x_{5} x_{3}, \\
\frac{\mathrm{d} x_{5}}{\mathrm{~d} t} & =p \frac{M}{J L_{r}}\left(x_{2} x_{3}-x_{1} x_{4}\right)-\frac{1}{J} T_{L}
\end{aligned}
$$

with

$$
\begin{aligned}
\lambda & =\frac{R_{s}}{\sigma} \frac{1}{L_{s}}+\frac{M^{2}}{\sigma} \frac{R_{r}}{L_{s} L_{r}^{2}}, ; \sigma=1-\frac{M^{2}}{L_{s} L_{r}}, \\
T_{r} & =\frac{L_{r}}{R_{r}}, \quad K=\frac{M}{\sigma L_{s} L_{r}} .
\end{aligned}
$$

We will drop the subscripts $r$ and $s$ since we will only use rotor fluxes $\left.\Phi_{r \alpha}, \Phi_{r \beta}\right)$ and stator currents $\left(i s \alpha, i_{s \beta}\right)$. Let

$$
x=\left(x_{1}, x_{2}, x_{3}, x_{4}, x_{5}\right)^{\top}=\left(i_{s \alpha}, i_{s \beta}, \Phi_{r \alpha}, P h i_{r \beta}, \Omega\right)^{\top} .
$$

Considering the vector of uncertain parameters

$$
\delta=\left[\begin{array}{l}
\delta_{1} \\
\delta_{2}
\end{array}\right]=\left[\begin{array}{l}
T_{L}-T_{L N} \\
R_{r}-R_{r N}
\end{array}\right]
$$

one can rewrite the equations of the asynchronous machine in the form suggested by

$$
\begin{aligned}
\dot{x}=f(x, \delta)+\sum_{i=1}^{2} & g_{i}(x, \delta) u_{i} \\
& =f(x)+\sum_{j=1}^{2} \delta_{j} f_{j}(x)+\sum_{i=1}^{2} g_{i}(x) u_{i}
\end{aligned}
$$

with

$$
\begin{gathered}
f(x)=\left[\begin{array}{c}
-\lambda x_{1}+\frac{k}{T_{r}} x_{3}+p k x_{4} x_{5} \\
-\lambda x_{2}+\frac{k}{T_{r}} x_{4}-p k x_{3} x_{5} \\
\frac{M}{T_{r}} x_{1}-\frac{1}{T_{r}} x_{3}-p x_{4} x_{3} \\
\frac{M}{T_{r}} x_{2}+p x_{3} x_{5}-\frac{1}{T_{r}} x_{4} \\
p \frac{M}{J L_{T}}\left(x_{2} x_{3}-x_{1} x_{4}\right)-\frac{T_{L}}{J}
\end{array}\right], \\
g(x)=\left[\begin{array}{cc}
\frac{1}{\sigma L_{s}} & 0 \\
0 & \frac{1}{\sigma L_{s}} \\
0 & 0 \\
0 & 0 \\
0 & 0
\end{array}\right], \\
\left.g_{1}(x) \quad g_{2}(x)\right]=\left[\begin{array}{cc}
-\frac{M}{\sigma L_{s} L_{r}^{2}} x_{1}+\frac{M}{\sigma L_{s} L_{r}^{2}} x_{3} \\
-\frac{M}{\sigma L_{s} L_{r}^{2}} x_{2}+\frac{M}{\sigma L_{s} L_{r}^{2}} x_{4} \\
\frac{M}{L_{r}} x_{1}-\frac{1}{L_{r}} x_{3} \\
\frac{M}{L_{r}} x_{2}-\frac{1}{T_{r}} x_{4} \\
0 \\
0 \\
0 \\
0 \\
-\frac{1}{J}
\end{array}\right], f_{2}(x)=\left[\begin{array}{c}
0 \\
0
\end{array}\right] .
\end{gathered}
$$

In order to increase the representation, we omit the dependence on $\mathrm{x}$. the derivatives of the outputs become

$$
\begin{aligned}
& \dot{y}_{1}=y_{2}+\delta_{1} L_{f_{1}} h_{1}, \\
& \dot{y}_{2}=L_{f}^{2} h_{1}+\delta_{2} L_{f_{2}} h_{1}+L_{g_{1}} L_{f} h_{1} u_{1}+L_{g_{2}} L_{f} h_{1} u_{2}, \\
& \dot{y}_{3}=y_{4}+\delta_{2} L_{f_{2}} h_{2}, \\
& \dot{y}_{4}=L_{f}^{2} h_{2}+\delta_{2} L_{f_{2}} L_{f} h_{2}+L_{g_{1}} L_{f} h_{2} u_{1}+L_{g_{1}} L_{f} h_{2} u_{1}, \\
& \dot{y}_{5}=L_{f} h_{3}+\delta_{2} L_{f_{2}} h_{3} .
\end{aligned}
$$

We can make a change of coordinates (diffeomorphism) using the estimated parameters $\hat{\delta}=\left[\hat{\delta}_{1}, \hat{\delta}_{2}\right]^{\top}$. The errors on these parameters are

$$
e_{\delta}=\left(\begin{array}{c}
e_{\delta_{1}} \\
e_{\delta_{2}}
\end{array}\right)=\left(\begin{array}{c}
\delta_{1}-\hat{\delta}_{1} \\
\delta_{2}-\hat{\delta}_{2}
\end{array}\right)
$$

Now we introduce the estimated parameters $\hat{\delta}$ in the new coordinates $z$

$$
\begin{aligned}
& z_{1}=y_{1}, \\
& z_{2}=y_{2}+\hat{\delta}_{1} L_{f_{1}} h_{1}, \\
& z_{3}=y_{3}, \\
& z_{4}=y_{4}+\hat{\delta}_{2} L_{f_{2}} h_{2}, \\
& z_{5}=y_{5} .
\end{aligned}
$$

The system (8) becomes

$$
\begin{aligned}
\dot{z}_{1} & =z_{2}+e_{\delta_{1}} L_{f_{1}} h_{1} \\
\dot{z}_{2} & =L_{f}^{2} h_{1}+\delta_{2} L_{f_{2}} L_{f} h_{1}+\frac{\mathrm{d} \hat{\delta}_{1}}{\mathrm{~d} t} L_{f_{1}} h_{1} \\
& +L_{g_{1}} L_{f} h_{1} u_{1}+L_{g_{2}} L_{f} h_{1} u_{2} \\
\dot{z}_{3} & =z_{4}+e_{\delta_{2}} L_{f_{2}} h_{2} \\
\dot{z}_{4} & =L_{f}^{2} h_{2}+\delta_{2} L_{f_{2}} L_{f} h_{2}+\frac{\mathrm{d} \hat{\delta}_{2}}{\mathrm{~d} t} L_{f_{2}} h_{2}+\hat{\delta}_{2} L_{f} L_{f_{2}} h_{2} \\
& +\hat{\delta}_{2} \delta_{2} L_{f_{2}}^{2} h_{2}+u_{1}\left(L_{g_{1}} L_{f} h_{2}+\hat{\delta}_{2} L_{g_{1}} L_{f_{2}} h_{2}\right) \\
& +u_{2}\left(L_{g_{2}} L_{f} h_{2}+\hat{\delta}_{2} L_{g_{1}} L_{f_{2}} h_{2}\right) \\
\dot{z}_{5} & \left.=L_{f} h_{3}+\delta_{2} L_{f_{2}} h_{3}\right) .
\end{aligned}
$$

Linearizing control, with the estimated parameters, is given by

$$
\left(\begin{array}{l}
u_{1} \\
u_{2}
\end{array}\right)=D\left(x, \hat{\delta}_{2}\right)^{-1}\left[\begin{array}{l}
-\hat{\xi}_{1}+v_{1} \\
-\hat{\xi}_{2}+v_{2}
\end{array}\right]
$$

where

$$
\begin{aligned}
\xi_{1} & =L_{f}^{2} h_{1}+\hat{\delta}_{2} L_{f_{2}} L_{f} h_{1}+\frac{\mathrm{d} \hat{\delta}_{1}}{\mathrm{~d} t} L_{f_{1}} h_{1}, \\
\xi_{2} & =L_{f}^{2} h_{2}+\hat{\delta}_{2} L_{f_{2}} L_{f} h_{2}+\frac{\mathrm{d} \hat{\delta}_{2}}{\mathrm{~d} t} L_{f_{2}} h_{2} \\
& +\hat{\delta}_{2} L_{f} L_{f_{2}} h_{2}+\hat{\delta}_{2}^{2} L_{f_{2}} h_{2}
\end{aligned}
$$


and

$D\left(x, \hat{\delta}_{2}\right)=$

$\left[\begin{array}{cc}L_{g_{a}} L_{f} h_{1} & L_{g_{b}} L_{f} h_{1} \\ L_{g_{a}} L_{f} h_{2}+\hat{\delta}_{2} L_{g_{a}} L_{f_{2}} h_{2} & L_{g_{b}} L_{f} h_{2}+\hat{\delta}_{2} L_{g_{b}} L_{f_{2}} h_{2}\end{array}\right]$.

New orders are stabilizing

$$
\begin{aligned}
& v_{1}=-k_{11}\left(z_{1}-z_{1 \mathrm{ref}}\right)-k_{12} z_{2}, \\
& v_{2}=-k_{21}\left(z_{3}-z_{3 \mathrm{ref}}\right)-k_{22} z_{4} .
\end{aligned}
$$

Control parameters $\left(k_{11}, k_{12}, k_{21}, k_{22}\right)$ are chosen so that

$$
K_{1}=\left[\begin{array}{cc}
0 & 1 \\
-k_{11} & -k_{12}
\end{array}\right], K_{2}=\left[\begin{array}{cc}
0 & 1 \\
-k_{21} & k_{22}
\end{array}\right]
$$

are asymptotically stable. The matrix gains $K_{1}$ and $K_{2}$ are given by (14). Then the tracking error is given by

$$
e=\left[\begin{array}{l}
e_{1} \\
e_{2} \\
e_{3} \\
e_{4}
\end{array}\right]=\left[\begin{array}{l}
z_{1}-z_{1 d} \\
z_{2}-z_{2 d} \\
z_{3}-z_{3 d} \\
z_{4}-z_{4 d}
\end{array}\right]
$$

Dynamics become

$$
\begin{aligned}
& \dot{e}_{1}=e_{2}+e_{\delta 1} L_{f_{1}} h_{1}, \\
& \dot{e}_{2}=-k_{11} e_{1}-k_{12} e_{2}+e_{\delta 2} L_{f_{2}} L_{f} h_{1}, \\
& \dot{e}_{3}=e_{4}+e_{\delta 2} L_{f_{2}} h_{2}, \\
& \dot{e}_{4}=-k_{21} e_{3}-k_{22} e_{4}+e_{\delta 2}\left(L_{f_{2}} L_{f} h_{2}+\hat{\delta}_{2} L_{f_{2}}^{2} h_{2}\right) .
\end{aligned}
$$

Or in matrix form

$$
\begin{aligned}
\left(\begin{array}{c}
\dot{e}_{1} \\
\dot{e}_{2} \\
\dot{e}_{3} \\
\dot{e}_{4}
\end{array}\right)= & {\left[\begin{array}{cccc}
0 & 1 & 0 & 0 \\
-k_{11} & -k_{12} & 0 & 0 \\
0 & 0 & 0 & 1 \\
0 & 0 & -k_{21} & -k_{22}
\end{array}\right]\left(\begin{array}{l}
e_{1} \\
e_{2} \\
e_{3} \\
e_{4}
\end{array}\right) } \\
+ & {\left[\begin{array}{cc}
L_{f_{1}} h_{1} & 0 \\
0 & L_{f_{2}} L_{f} h_{1} \\
0 & L_{f_{2}} h_{2} \\
0 & L_{f_{2}} L_{f} h_{2}+\dot{\delta}_{2} L_{f_{2}}^{2} h_{2}
\end{array}\right]\left(\begin{array}{l}
e_{\delta_{1}} \\
e_{\delta_{2}}
\end{array}\right) . }
\end{aligned}
$$

In the condensed form we have

$$
\dot{e}=K e+W\left(z, z h a t z \text { delta } a_{2}\right) e_{\delta}
$$

with

$$
\begin{gathered}
e_{\delta}=\left[e_{\delta_{1}}, e_{\delta_{2}}\right]^{\top} ; e=\left[e_{1}, e_{2}, e_{3}, e_{4}\right]^{\top}, \\
K=\operatorname{bloc} \operatorname{diag}\left(K_{1}, K_{2}\right)
\end{gathered}
$$

and

$$
W(z, \hat{\delta})=\left[\begin{array}{cc}
L_{f_{1}} & 0 \\
0 & L_{f_{2}} L_{f} h_{1} \\
0 & L_{f_{2}} h_{2} \\
0 & L_{f_{2}} L_{f} h_{2}+\hat{\delta}_{2} L_{f_{2}}^{2} h_{2}
\end{array}\right]
$$

\section{NATURAL DYNAMICS COMPLETE OBSERVER}

We develop a nonlinear control law with a comprehensive observer. According to [1] we use the complete model of the machine, which implies a relative degree one and two of the output. We develop a control law that controls the torque and flux of the machine (see Fig. 1). We use an estimator with zero gain observers. The control uses only quantities estimated.

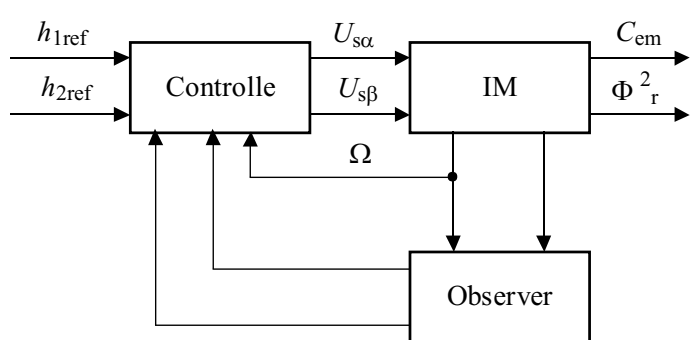

Fig. 1. Nonlinear control with dynamic natural observer $\boldsymbol{n}$

$$
\begin{aligned}
Y(x) & =\left[\begin{array}{c}
\hat{h}_{1}(x) \\
\hat{h}_{2}(x)
\end{array}\right]=\left[\begin{array}{c}
p \frac{M}{L_{r}}\left(\hat{x}_{2} \hat{x}_{3}-\hat{x}_{1} \hat{x}_{4}\right) \\
\hat{x}_{3}^{2}+\hat{x}_{4}^{2}
\end{array}\right], \\
\hat{h}_{3}(x) & =2 \frac{M}{T_{r}}\left(\hat{x}_{3} \hat{x}_{1}+\hat{x}_{4} \hat{x}_{2}\right) .
\end{aligned}
$$

\subsection{Observer development}

The machine model that uses the model with four streams flux it self particularly well to find a Lyapunov function which can show the stability of the estimator. We choose this representation only for easier demonstration of the stability of the observer. The current estimates could be obtained easily with a transformation matrix from invariant flux estimated.

$$
\begin{gathered}
\dot{\hat{X}}=A_{2}(\Omega) \hat{\bar{X}}+B u, \\
\hat{\bar{X}}=\left[\hat{\Phi}_{s \alpha}, \hat{\Phi}_{s \beta}, \hat{\Phi}_{r \alpha}, \hat{\Phi}_{r \beta}\right]^{\top}, \\
A(\Omega)=-R L^{-1}+p \Omega\left[\begin{array}{cc}
0 I & 0 I \\
0 I & J
\end{array}\right], \\
B=\left[\begin{array}{cccc}
1 & 0 & 0 & 0 \\
0 & 1 & 0 & 0
\end{array}\right], I=\left[\begin{array}{cc}
1 & 0 \\
0 & 1
\end{array}\right], J=\left[\begin{array}{cc}
0 & 1 \\
-1 & 0
\end{array}\right], \\
R=\left[\begin{array}{cc}
R_{s} I & 0 I \\
0 I & R_{r} I
\end{array}\right], L=\left[\begin{array}{ll}
L_{s} I & M I \\
M I & L_{r} I
\end{array}\right] .
\end{gathered}
$$

We define the estimation errors

$$
\bar{e}=\left[e_{4}, e_{5}, e_{6}, e_{7}\right] .
$$

Let us choose the function of Lyapunov $V_{1}$ following [1]

$$
V_{1}=\frac{1}{2} \bar{e}^{\top} R^{-1} \bar{e}
$$

Its derivative is equal to

$$
\begin{aligned}
& \dot{V}_{1}=\frac{1}{2} \bar{e}^{\top}\left(-\left(R L^{-1}\right)^{\top} R^{-1}+p \Omega\left[\begin{array}{cc}
0 I & 0 I \\
0 I & J
\end{array}\right]^{\top} R^{-1}\right. \\
& \left.+R^{-1} R L^{-1}+R^{-1} p \Omega\left[\begin{array}{cc}
0 I & 0 I \\
0 I & J
\end{array}\right]\right) \bar{e}=-\bar{e}^{\top}\left(L^{-1}\right) \bar{e}
\end{aligned}
$$

with $L^{-1}$ positive definite $\left(\operatorname{det} L=L_{s} L_{r} \sigma ; 0<\sigma<1\right)$. This proves the stability of the observer. 


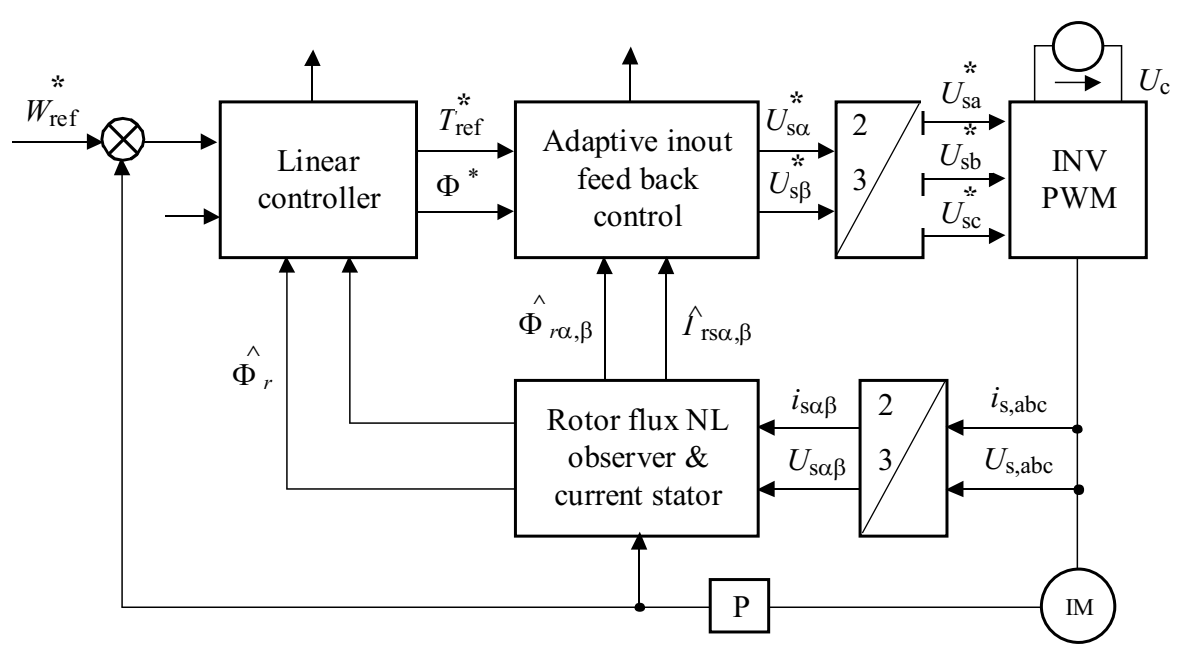

Fig. 2. Decoupled with AIOL

\subsection{Control development with observer}

We can develop the control law using the observer and the mechanical equation

$$
\left[\begin{array}{c}
\dot{\hat{\bar{X}}} \\
\Omega=x_{5}
\end{array}\right]=\left[\begin{array}{c}
A(\Omega) \hat{\bar{X}} \\
\frac{1}{L}\left(p \frac{M}{L_{r}}\left(x_{3} x_{2}-x_{4} x_{1}\right)-C_{\mathrm{res}}\right)
\end{array}\right]+\left[\begin{array}{c}
B \\
0
\end{array}\right] u .
$$

On this model we have the system of tracking errors of torque and flux following

$$
\left[\begin{array}{c}
\dot{e}_{1} \\
\dot{e}_{2} \\
\dot{e}_{3}
\end{array}\right]=\left[\begin{array}{c}
L_{f} \hat{h}_{1} \\
L_{f} \hat{h}_{2} \\
L_{f} \hat{h}_{3}
\end{array}\right]+\left[\begin{array}{c}
L_{g} \hat{h}_{1} \\
0 \\
L_{g} \hat{h}_{3}
\end{array}\right] u-\left[\begin{array}{c}
\dot{h}_{1 \mathrm{ref}} \\
\dot{h}_{2 \mathrm{ref}} \\
\dot{h}_{3 \mathrm{ref}}
\end{array}\right] .
$$

To obtain a further output flux and torque, choose

$$
u=\left[\begin{array}{c}
L_{g} \hat{h}_{1} \\
L_{g} \hat{h}_{3}
\end{array}\right]^{-1}\left[\begin{array}{c}
-L_{f} \hat{h}_{1}-k_{1} e_{1}+\hat{h}_{1 \mathrm{ref}} \\
-L_{f} \hat{h}_{3}-k_{3} e_{3}+\hat{h}_{3 \mathrm{ref}}-e_{2}
\end{array}\right] .
$$

By applying the command (30) we obtain the following system errors stability of the control.

$$
\begin{aligned}
{\left[\begin{array}{c}
\dot{e}_{1} \\
\dot{e}_{2} \\
\dot{e}_{3}
\end{array}\right] } & =\left[\begin{array}{ccc}
-k_{1} & 0 & 0 \\
0 & 0 & 0 \\
0 & -1 & -k_{3}
\end{array}\right]\left[\begin{array}{c}
e_{1} \\
e_{2} \\
e_{3}
\end{array}\right]+\left[\begin{array}{c}
0 \\
L_{f} \hat{h}_{2}-h_{2 \mathrm{ref}} \\
0
\end{array}\right] \\
\dot{\bar{e}} & =R L^{-1} \bar{e}
\end{aligned}
$$

with

$$
L_{f} \hat{h}_{2}=\hat{h}_{3}-\frac{2}{T_{r}} \hat{h}_{2}-\dot{h} 2 \mathrm{ref} .
$$

Choosing

$$
h_{3 \mathrm{ref}}=\frac{2}{T_{r}} \hat{h}_{2}+\dot{h}_{2 \mathrm{ref}}-k_{2} e_{2}
$$

and

$$
L_{f} \hat{h}_{2}=e_{3}+\dot{h}_{22 \mathrm{ref}}-k_{2} e_{2}
$$

we obtain the system of tracking errors

$$
\left[\begin{array}{c}
\dot{e}_{1} \\
\dot{e}_{2} \\
\dot{e}_{3}
\end{array}\right]=\left[\begin{array}{ccc}
-k_{1} & 0 & 0 \\
0 & -k_{2} & 1 \\
0 & -1 & -k_{3}
\end{array}\right]\left[\begin{array}{l}
e_{1} \\
e_{2} \\
e_{3}
\end{array}\right]
$$

The Lyapunov function $V_{2}$ with its derivative along the trajectories of the system ensures the asymptotic

$$
\begin{aligned}
& V_{2}=\frac{1}{2}\left(e_{1}^{2}+e_{2}^{2}+e_{3}^{2}\right), \\
& \dot{V}_{2}=-k_{1} e_{1}^{2}-k_{2} e_{2}^{2}-k_{3} e_{3}^{2} .
\end{aligned}
$$

We can conclude on the overall stability of the entire control and observer using Lyapunov function candidate $V$ with its derivative

$$
\begin{aligned}
& V=V_{1}+V_{2} \\
& \dot{V}=-k_{1} e_{1}^{2}-k_{2} e_{2}^{2}-k_{3} e_{3}^{2}-\bar{e}^{\top}\left(L^{-1}\right) \bar{e}
\end{aligned}
$$

with $V>0$ and $\dot{V}<0$ we can deduce the stability of the observer and control using a complete model of the motor and a complete observer.

\section{SIMULATION RESULTS}

The proposed PI for controlling the speed of IM with AIOL decoupling and flux NLO was designed for $1.5 \mathrm{kw}$ drive is represented in Fig. 2. We interpret some simulation results with parameter variations of $50 \%$ on the rotor resistance ( $\mathrm{Rr}$ ) and the variation of torque (TL). We conduct a comparison of three cases in order to evaluate the performance of adaptive control with or without the use of a flux observer, these are:

1) Nonlinear control sets (without adaptation).

This control is developed based on the nominal model $\left(R_{r N}, T_{L N}\right)$ applied to the model characterized by parameters $R_{r}$ and $T_{L}$ show a variation of $50 \% R_{r}$ at $t=2 \mathrm{~s}$ to $t=5 \mathrm{~s}$ and a torque variation $T_{L} 10 \mathrm{~N}$.m equal to $t=(1.5 \mathrm{~s}, 2.5 \mathrm{~s})$ and $-10 \mathrm{~N} . \mathrm{m}$ at $t=(5.5 \mathrm{~s}, 6.5 \mathrm{~s})$. 

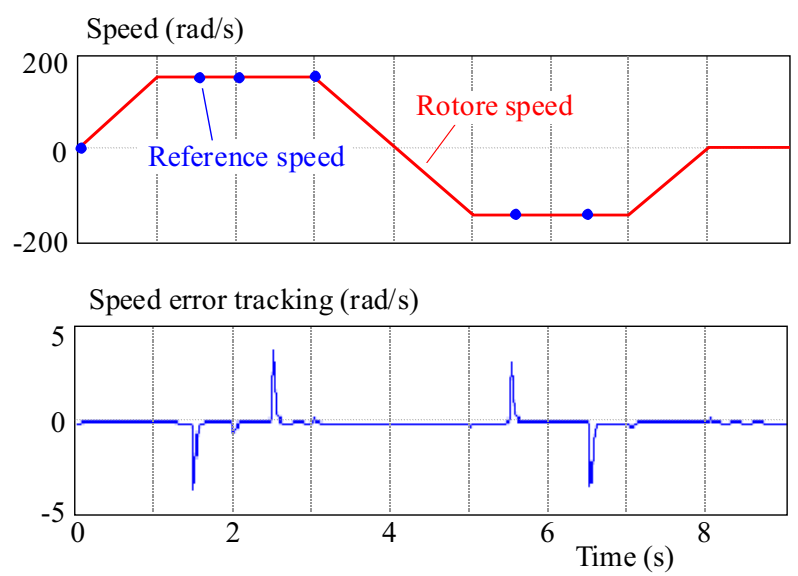

Fig. 3. Mechanical speed and speed error
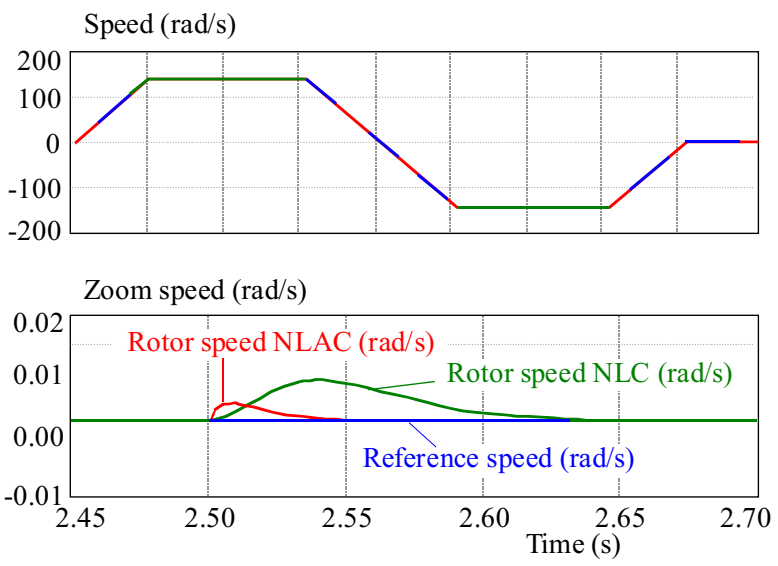

Fig. 5. Mechanical speed control

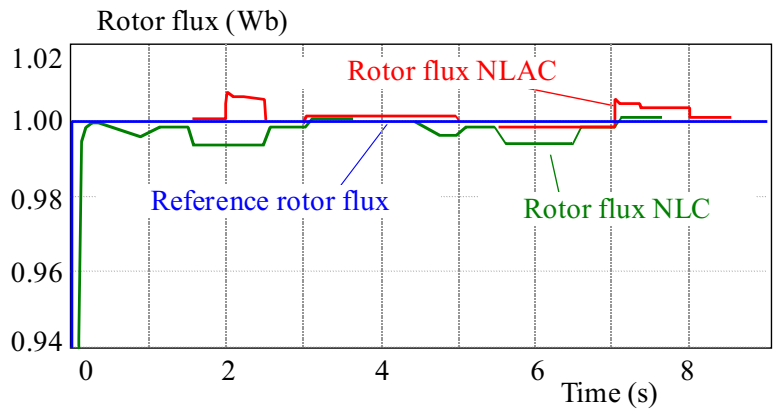

Fig. 7. Rotor flux

\section{2) Nonlinear adaptive control.}

3) Nonlinear control with adaptive nonlinear observer.

This control law is developed similarly to the previous one using a robust nonlinear observer flux and current. We have chosen to adapt the following gains: $\Gamma=(1000,1000)^{\top}$ see equation $(38)$, with the weighting $Q=(0.005 ; 1 ; 0.001)$ see equation $(35)$. The results found are as follows

- According to the Figs. 3 and 4, we noted that despite the application of the load torque and the rotor resistance variations, speed and flux return to their initial values along very short time. We confirm that the speed and flux perfectly follow their references.
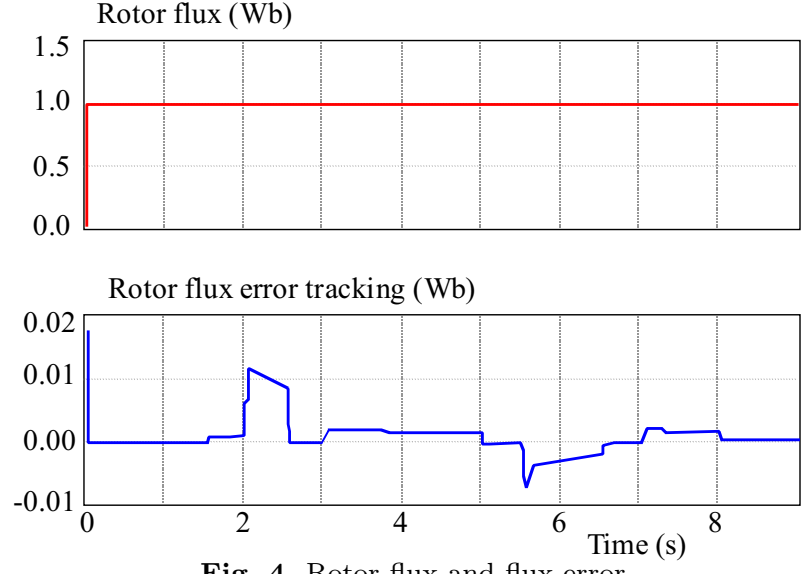

Fig. 4. Rotor flux and flux error

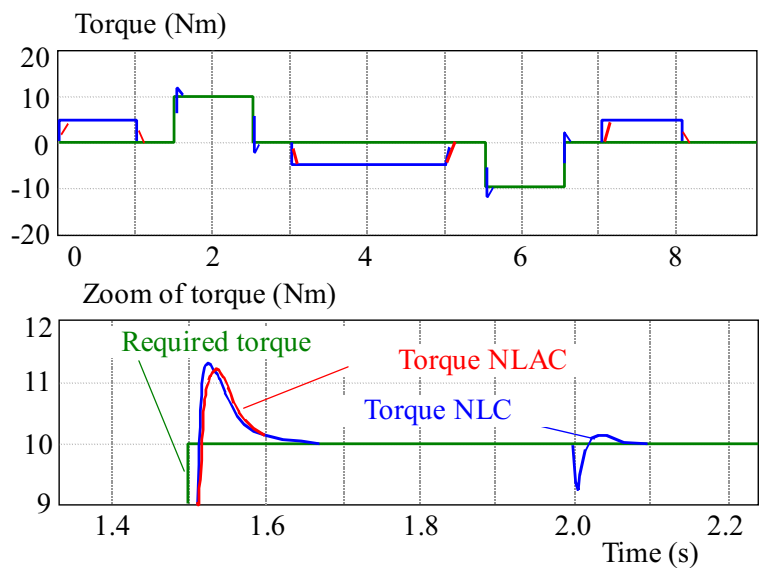

Fig. 6. Torques

- In the comparison between the fixed nonlinear control and the adaptive nonlinear control without observer (case 1 and case 2), we base ourselves on the Figs. 5 to 7 which show the speed,torques and flux. We notice that the presence of the parametric variations involves a coupling between flux and torque for the fixed nonlinear control.

- Knowing that the errors of continuation speed are due to the presence of a variable load torque of $10 \mathrm{~N}$.m to $t=(1.5$ to 2.5$) \mathrm{s}$ and $-10 \mathrm{~N} . \mathrm{m}$ to $t=(5.5$ to 6.5$) \mathrm{s}$, we note that only the adaptive nonlinear control allows a good continuation of flux and that it ensures, with effectiveness, the limitation of current.

- Our second comparison between the adaptive nonlinear control and the adaptive nonlinear control with observer (case 2 and case 3), shows the interest to adapt the model used by the observer. The use of an observer allows primarily a better continuation speed, flux and torque (see Figs. 8-11), then giving a good limitation of the current.

\section{CONCLUSION}

In this article we developed an adaptive nonlinear control which takes account of the parametric variations of rotor resistance and the load torque. The latter makes it possible to ensure an effective limitation of current.

Theoretically we established a proof of stability of the adaptive nonlinear control in the case or flux is measured. 

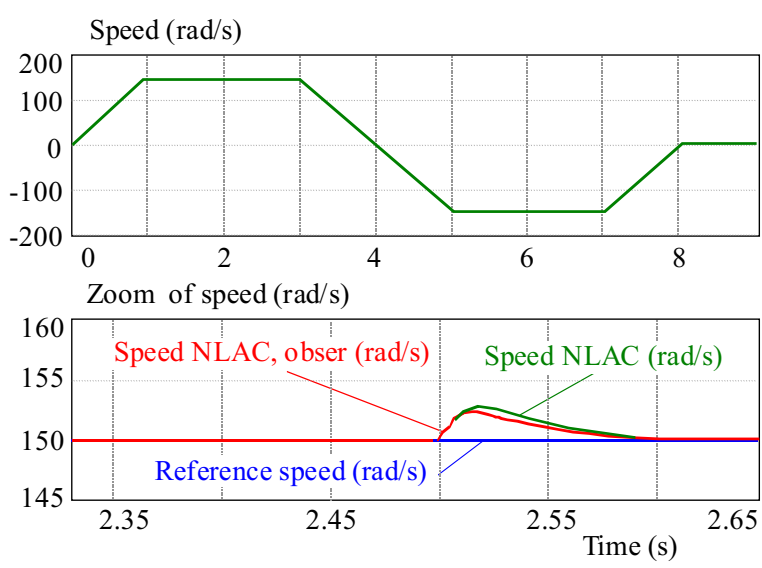

Fig. 8. Mechanical speed
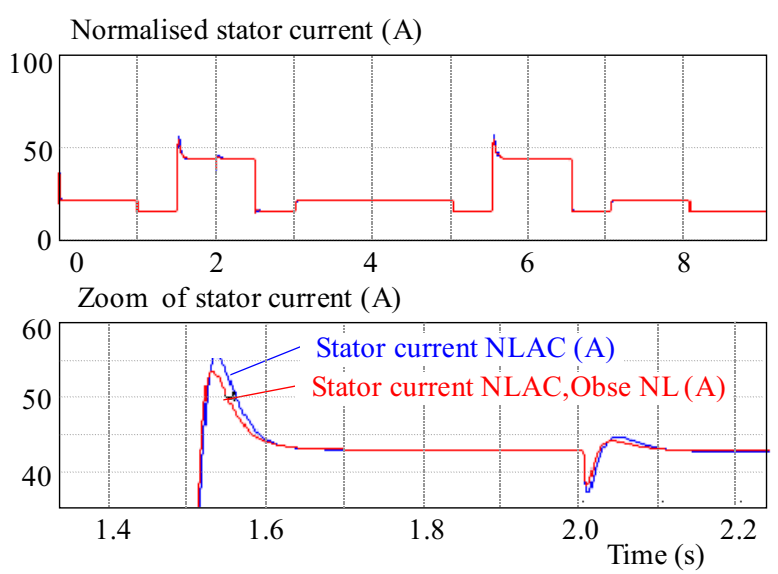

Fig. 10. Stator current

Table 1.

\begin{tabular}{lcl}
\hline Designation & Parameter & value \\
\hline Rotor resistance & $R$ & $3.81 \Omega$ \\
Stator resistance & $R$ & $4.85 \Omega$ \\
Mutual inductance & $M$ & $0.258 \mathrm{H}$ \\
Stator inductance & $L$ & $0.274 \mathrm{H}$ \\
Rotor inductance & $L$ & $0.274 \mathrm{H}$ \\
Rotor inertia & $J$ & $0.031 \mathrm{Kgm}^{2}$ \\
Pole pair & $p$ & 2 \\
Viscous frict. Coef. & $f$ & $0.0114 \mathrm{Nm} / \mathrm{rd} / \mathrm{s}$ \\
Mechanical power & $P$ & $1.5 \mathrm{~kW}$ \\
Nominal voltage & $V$ & $220 \mathrm{~V}$ \\
Nominal current & $I$ & $3 \mathrm{~A}$ \\
Nominal speed & $n$ & $1450 \mathrm{tr} / \mathrm{mn}$ \\
\hline
\end{tabular}

However in reality we must use an observer because flux is not accessible to measurement. The studies of simulation made it possible to show a better performance when an observer of flux is used.

\section{APPENDIX}

\section{Induction motor parameters}

The induction motor used in this system is a threephase, Y-connected, four poles, $1.5 \mathrm{Kw}, 50 \mathrm{~Hz}, 220 \mathrm{~V} / 3 \mathrm{~A}$
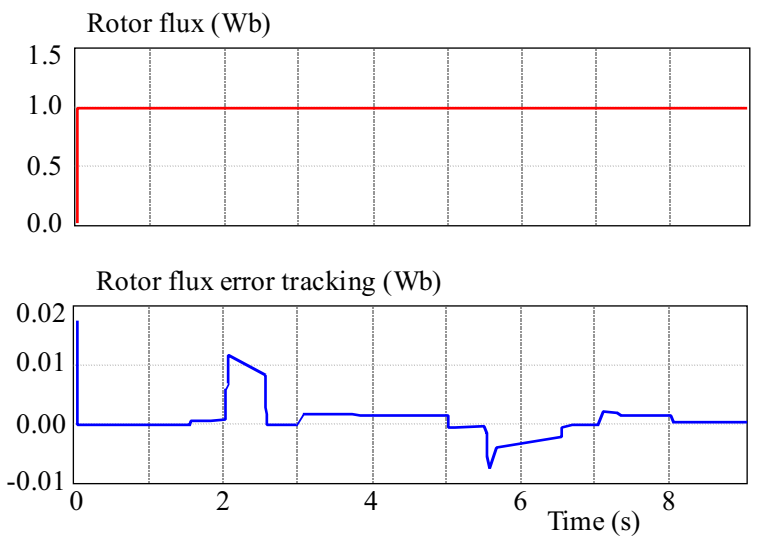

Fig. 9. Rotor flux
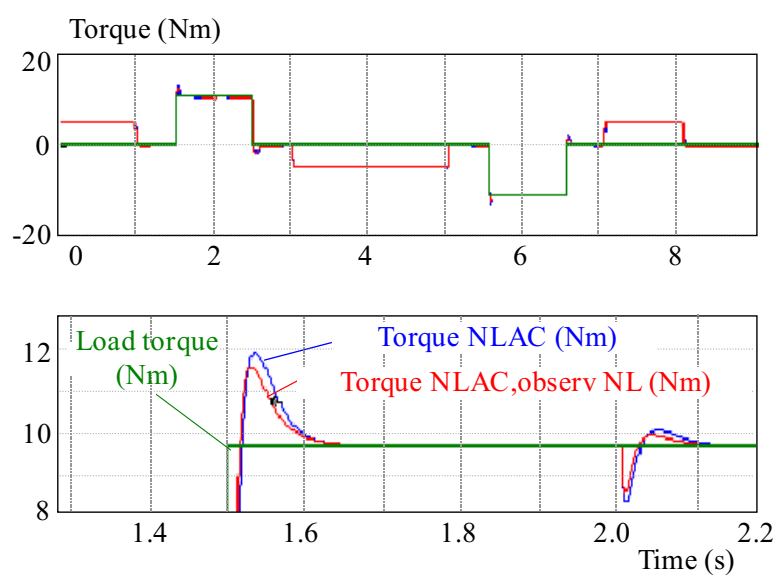

Fig. 11. Torques

type. The nominal values of the motor used in this simulation are given in the Table 1.

\section{Lie derivation estimation}

$$
\begin{aligned}
L_{f} \hat{h}_{1} & =-p \frac{M}{L_{r}}\left[p x_{5}\left(\hat{x}_{3} \hat{x}_{1}+\hat{x}_{4} \hat{x}_{2}\right)\right. \\
& \left.+\left(\lambda+\frac{1}{T_{r}}\right)\left(\hat{x}_{3} \hat{x}_{2}+\hat{x}_{4} \hat{x}_{1}\right)+p x_{5} K\left(\hat{x}_{3}^{2}+\hat{x}_{4}^{2}\right)\right], \\
L_{f} \hat{h}_{2} & =\frac{2}{T_{r}}\left[M\left(\hat{x}_{3} \hat{x}_{1}+\hat{x}_{4} \hat{x}_{2}\right)-\left(x_{3}^{2}+x_{4}^{2}\right)\right], \\
L_{f} \hat{h}_{3} & =\frac{2 M^{2}}{T_{r}^{2}}\left(\hat{x}_{1}^{2}+\hat{x}_{2}^{2}\right)-2 M\left(\frac{\lambda}{T_{r}}+\frac{1}{T_{r}^{2}}\right)\left(\hat{x}_{3} \hat{x}_{1}+\hat{x}_{4} \hat{x}_{2}\right) \\
& +\frac{2 M p x_{5}}{T_{r}}\left(\hat{x}_{3} \hat{x}_{2}-\hat{x}_{4} \hat{x}_{1}\right)+\frac{2 K M}{T_{r}^{2}}\left(\hat{x}_{3}^{2}+\hat{x}_{4}^{2}\right), \\
L_{g} \hat{h}_{1} & =\left[-p K \hat{x}_{4},-p K \hat{x}_{3}\right], \\
L_{g} \hat{h}_{3} & =\left[2 R_{r} K \hat{x}_{3}, 2 R_{r} K \hat{x}_{4}\right] .
\end{aligned}
$$

\section{REFERENCES}

[1] MARINO, R.-PERESADA, S.-TOMEI, P. : Adaptative Output Feedback Control of Current-Fed Induction Motors, $12^{\text {th }}$ IFAC World Congress, 1993. 
[2] MARINO, R.-TOMEI, P.: Nonlinear Control Design: Geometric, Adaptive and Robust, Prentice Hall, 1995.

[3] AMOR, L. B.: Internat. J. Adaptive Control and Signal Processing 7 (1993).

[4] GRCAR, B.-CAFURA, P.-ZNIDARIC, M.-GAUSCH Nonlinear Control of Synchronous Servo Drives: IEEE Trans. on Control Systems Technology 4 No. 2 (March 1996).

[5] ZRIBI, M.-CHIASSON, J.: IEEE Trans. on Autom. Control AC-36 (1991), 620-625.

[6] GRENIER, D.-DESSAINT, L.-AKHRIF, A. O.-BONNASSIEUX. Y.-PIOUFFE, B.: Experimental Nonlinear Torque Control of a PMSM using Saliency, IEEE Trans. on Industrial Electronics 44 (Oct 1997), 680-686.

[7] MARINO, R.-KANELLAKOPOULOS, I.-KOKOTOVIC, P. : Adaptive Traking for Feedback Linearizable SISO Systems, Proc. $28^{\text {the }}$ IEEE Conf. on Decision and Control, Dec 1989, pp. 1002-1007.

[8] MARINO, R.-PERESADA, S.-VAligi, P.: Adaptive Partial Feedback Linearization of Induction Motors, Proc. $29^{\text {th }}$ IEEE conf. On Decision and Control, Dec 1990, pp. 3313-3318.

[9] KADDOURI, A.: Study of an Adaptive Non-Linear Control of a Synchronous Permanent Magnet Machine, Thesis of Doctorate in Electrical Engineering, Laval University, Quebec, Canada, 2000.

[10] KABACHE, N.-CHETATE, B.: Adaptive Non-Linear Control of an Asynchronous Machine by using Networks of Artificial Neurons Trailed in Real Times, Re-Examined Renewable Energies, Proceedings of the $1^{\text {st }}$ International Conference on the Energy Effectiveness, 2003, pp. 457-463.

[11] CHETATE, B.-KABACHE, N.-BRADAI, R.: Adaptive Control of Induction Motor with Unknown Motor Resistance, AMSE Journal, Advanced C (2007), 83-93.

[12] QUASSAID, M.-CHERKAOUI, M.-ZIDANI, Y.: A Nonlinear Speed Control for a PM Synchronous Motor using an Adaptive Back Stepping Control Approach, IEEE, ICIT, 04 IEEE International Conference,, vol. 3, 2004, pp. 1287-1292.

[13] KADDOURI, A.-GHRIBI, M.-AKHRIF, O.-LE-HUY, H. : Adaptive Nonlinear Control of an Electric Motor, Journal of Applied Mathematics Sciences 2 No. 52 (2008), 2557-2568.

[14] MERABET, A.: Non-Linear Control with Predictive Model for an Asynchronous Machine, Thesis of Doctorate in Engineering, University of Quebec, Canada, 2007.

[15] TAYLOR, D. G.-KOKOTOVIC, P. V.-MARINO, R.KANLLAKOPOULOS. : Adaptive Regulation of Nonlinear Sys- tems with Unmodeled Dynamics, IEEE Trans. on Automatic Control 34 (Nov 1989), 405-412.

Received 11 January 2011

Abderrahim Bentaallah was born in Sidi Bel-Abbes (Algeria) in 1965; He received his BS degree in Electrical ingeneering from Sidi Bel-Abbes University (Algeria) in 1991, the MS degree from the same University in 2005 and the $\mathrm{PhD}$ degree from The Electrical Engeneering Institute of University of Sidi Bel-Abbes (Algeria) in 2009. He is currently Professor of electrical engineering in this University. He is a member in (ICEPS). His research interests are robust control of electrical machines.

Ahmed Massoum was born in 1959 in M'sirda Fouaga, Tlemcen, Algeria. He received his BS degree in electrical engineering from the Electrical Engineering Institute (INELEC) of Boumerdes 1985 and the MS degree from the Electrical Engineering Institute of Sidi Bel-Abbes University in 2004 where he is currently Professor of electrical engineering. He is a member in Intelligent Control Electrical Power System Laboratory (ICEPS).

Farid Benhamida received the BS degree from Djilali Liabes University, Sidi Bel-Abbes, Algeria, in 1999, the MS degree from University of technology, Bagdad, Iraq, in 2003, and the $\mathrm{PhD}$ degree from Alexandria University, Alexandria, Egypt, in 2006, all in electrical engineering. Presently, he is an Assistant Professor in the Electrical Engineering Department and a Research Scientist in the IRECOM laboratory (Laboratoire Interaction rseaux lectriques Convertsisseurs Machines), Faculty of engineering, Djilali Liabes University.

Abdelkader Meroufel was born in Sidi Bel-Abbes (Algeria) in 1954. He received his BS degree in Electrical ingeneering from the USTOran (Algeria) in 1979, the MS degree from the same University (USTO) in 1990 and the PhD degree from The Electrical Engeneering Institute of University of Sidi Bel-Abbes (Algeria) in 2004. He is currently Professor of electrical engineering in this University. He is a member of ICEPS (Intelligent Control Electrical Power System) Laboratory. His research interests are robust control of electrical machines. 